INPLASY

PROTOCOL

To cite: Tang et al. Chinese herb injection combined with chemotherapy for advanced colorectal cancer: A protocol for Bayesian network metaAnalysis. Inplasy protocol 202130089. doi: 10.37766/inplasy2021.3.0089

Received: 24 March 2021

Published: 24 March 2021

Corresponding author:

Shengnan Tang

450606486@qq.com

Author Affiliation:

Gansu University of Chinese Medicine

Support: Yes.

Review Stage at time of this submission: Preliminary searches.

Conflicts of interest:

None declared.

\section{Chinese herb injection combined with chemotherapy for advanced colorectal cancer: A protocol for Bayesian network meta-Analysis}

Tang, SN1; Teng, R²; Zhao, DS3; Nie, GY4; Dong, ZP5; Zhang, WJ6.

Review question / Objective: The aim of this study was to assess the clinical efficacy and safety of different Chinese herb injections combined with chemotherapy regimen for CRCs and to provide evidence for rational selection of $\mathrm{CHIs}$ using network meta-analysis.

Condition being studied: Colorectal cancer (CRC) is the third most commonly diagnosed cancer in the worldwide. Surgical resection is the primary treatment modality for stage I-III colorectal cancer, with systemic chemotherapy or radiotherapy. Chinese herb injections (CHIs) have been proven to be effective against a variety of cancers.

INPLASY registration number: This protocol was registered with the International Platform of Registered Systematic Review and Meta-Analysis Protocols (INPLASY) on 24 March 2021 and was last updated on 24 March 2021 (registration number INPLASY202130089).

\section{INTRODUCTION}

Review question / Objective: The aim of this study was to assess the clinical efficacy and safety of different Chinese herb injections combined with chemotherapy regimen for CRCs and to provide evidence for rational selection of CHls using network meta-analysis.

Condition being studied: Colorectal cancer (CRC) is the third most commonly diagnosed cancer in the worldwide. Surgical resection is the primary treatment modality for stage I-III colorectal cancer, 
with systemic chemotherapy or radiotherapy. Chinese herb injections (CHIs) have been proven to be effective against a variety of cancers.

\section{METHODS}

Participant or population: Patients must be cytologically or pathologically confirmed as having CRC at a clinically advanced stage. And other malignancies or non-primary CRCs are not included. The patient must be an adult (age $\geq 18$ years). However, there will no restriction on gender, race, economic and education status.

Intervention: In the advanced CRC of experimental group, must be treated with chemotherapy treatment combined with at least one Chinese herb injection therapy. There will no restrictions on the types, dosages, and duration of chemotherapy drugs and Chinese herb injection. In the control group, advanced CRC treated with the same chemotherapy treatment as intervention group in the same original research.

Comparator: In the control group, advanced CRC treated with the same chemotherapy treatment as intervention group in the same original research.

Study designs to be included: We plan to included randomized controlled trials (RCTs) compared the efficacy and safety between Chinese herb injection combined with chemotherapy and chemotherapy for advanced CRC. Qualitative studies, case control studies, observational studies, and case reports will be excluded. If the data cannot be obtained by contacting the author, the studies reported in abstract form that without presenting the outcome will be excluded.

Eligibility criteria: We plan to included randomized controlled trials (RCTs) compared the efficacy and safety between Chinese herb injection combined with chemotherapy and chemotherapy for advanced CRC. Patients must be cytologically or pathologically confirmed as having CRC at a clinically advanced
stage.In the advanced CRC of experimental group, must be treated with chemotherapy treatment combined with at least one Chinese herb injection therapy.

Information sources: A systematic search will be performed in eight public domain electronic databases from inception to December 2020. The databases are as follows: PubMed, Cochrane Library, Embase, Web of Science, Chinese National Knowledge Infrastructure, WanFang database, China Biology Medicine Database and Chongqing VIP Chinese Scientific Journals Full-text Database.

Main outcome(s): The treatment response rate; Overall survival at 0.5 -year $(0.5-0 S)$, one-year (1-OS), three years (3-OS), and five years (5-OS).

Additional outcome(s): The local tumor treatment response: complete response, partial response, progressive disease, and stable disease [25]; Disease progression at one year confirmed radiologically or biopsy proven; if data are available, will plus three years or five years; Disease recurrence at one year confirmed radiologically or biopsy proven; if data are available, will plus three years or five years; Quality of life evaluated using any validated assessment tool; Immune function: the levels of CD3+, CD4+, CD8+, CD4+/CD8+, NK cells, IL-2, $\lg A, \lg G$ and IgM;Adverse effect rates: decreases in leukocytes and platelets, nausea and vomiting, debilitation, diarrhea and stomachache.

Quality assessment / Risk of bias analysis: The Cochrane Risk of Bias (RoB) Tool for RCTs will be used to evaluate the risk of bias of each included RCT and quasirandomized trial [26]. The RoB tool has seven domains and each aspect is judged as three levels: high risk of bias, unclear risk of bias, and low risk of bias. All domains will be considered, including sequence generation and allocation sequence concealment (selection bias), blinding of participants and personnel (performance bias), blinding of outcome assessment (detection bias), incomplete 
outcome data (attrition bias), selective reporting (reporting bias), and other biases considered relevant to the review topic. Two reviewers will independently assess the quality of included studies. Any disagreements will be resolved by consensus or with a third reviewer.

Strategy of data synthesis: Firstly, we will perform pairwise meta-analysis using a random-effects model in Stata 14.0 software for every direct treatment comparison with at least two primary studies. About the study effect size, we will calculate the odds ratios for binary outcomes and standardized mean differences for continuous outcomes, with $95 \%$ confidence interval. And we will estimate the heterogeneity for each comparison by Chi-squared test and 12 test. $12 \leq 25 \%$ is considered to reflect low heterogeneity, $25 \%$ high heterogeneity. We will prespecifies meta-regression analyses to explore the sources of heterogeneity. If heterogeneity is high, we will perform a subgroup analysis. And if cannot perform subgroup analysis, We will performer a descriptive analysis.Secondly, in order to determine if a NMA is feasible, we will initially generate and assesses the network diagrams. Then a random-effects Bayesian NMA will be performed by using the $R$ software.

Subgroup analysis: If heterogeneity is high, we will perform a subgroup analysis. And if cannot perform subgroup analysis, We will performer a descriptive analysis.

Sensitivity analysis: The assessment of statistical heterogeneity in the entire network will be based on the magnitude of the heterogeneity variance parameter estimated from the multivariate metaanalysis. And when $12>60 \%$, the certainty of the evidence will be downgraded for inconsistency. We will use the "design-bytreatment" interaction model to inferred about the presence of inconsistency from any source in the entire network based on 12. Like the pairwise meta-analysis, when we found important heterogeneity and/or inconsistency, we will explore the possible sources for primary outcomes. If sufficient studies are available, we will performe multivariate meta-analyses for subgroups and sensitivity analyses by using potential effect modifiers as possible sources of inconsistency and/or heterogeneity.

Language: None restriction.

Country(ies) involved: China.

Keywords: Chinese herb injection, Chemotherapy, Colorectal cancer, Protocol, Network meta-analysis.

Contributions of each author:

Author 1 - Shengnan Tang - Methodology; Project administration; Writing-original draft.

Email: 450606486@qq.com

Author 2 - Ran Teng - Resources; Writingreview \& editing.

Email: 136369968@qq.com

Author 3 - Dangsheng Zhao - Funding acquisition.

Email: zhds69@126.com

Author 4 - Guiyuan Nie - Software.

Email: 1037209735@qq.com

Author 5 - Zhuanping Dong - Software.

Email: 1951154574@qq.com

Author 6 - Wenjun Zhang - Project administration; Resources; Supervision; Validation; Writing-review \& editing.

Email: 1272025508@qq.com 